\title{
On Superstatistical Multiplicative-Noise Processes
}

\author{
Sílvio M. Duarte Queirós* \\ Centro Brasileiro de Pesquisas Físicas, Rua Dr. Xavier Sigaud, 150, 22250-250 Rio de Janeiro - RJ, Brazil
}

Received on 28 September, 2007

\begin{abstract}
In this article we analyse the long-term probability density function of non-stationary dynamical processes with time varying multiplicative noise exponents which are enclosed inwards the Feller class of processes. The update in the value of the exponent occurs in the same conditions as presented by BECK and COHEN for superstatistics. Moreover, we are able to provide a dynamical scenario for the emergence of a generalisation of the Weibull distribution previously introduced.
\end{abstract}

Keywords: Multiplicative noise; Superstatistics; Generalized Weibull distribution

\section{INTRODUCTION}

The description within a physical context of driven nonequilibrium complex systems has frequently been made by considering that their dynamical behaviour is characterised by spatial-temporal fluctuations of some parameter, $\tilde{\beta}$. Usually, this parameter has been considered to be the (inverse) temperature, the dissipation of energy in turbulent flows, the amplitude of Gaussian white noise, the local mean-reverting value or the local variance. As an example, we mention the standard case of a Brownian particle diffusing along an inhomogeneous medium in which temperature (hence diffusion "constant") fluctuates in both space and time. In this approach, as it can be understood, there are two important time scales: the scale in which the dynamics is able to reach a stationary state (assuming a fixed value for parameter $\tilde{\beta}$ ), and the scale at which the fluctuating parameter evolves. A particular case to consider is when these two time scales are clearly separated, specifically, when the time needed for the system to reach stationarity (considering a predetermined $\tilde{\beta}$ ) is much smaller than the scale at which that parameter changes. In the long-term, the non-equilibrium system is described by the superposition of different local dynamics at different time intervals that was coined by BECK and COHEN as superstatistics or "statistics of statistics" $[1,2]$. Frequently, systems that are characterised as "superstatistical" exhibit non-Gaussian distributions with kurtosis excess, or distributions with non-exponential decay. In addition, superstatistical systems present a parameter, $\tilde{\beta}$, that fluctuates on a large scale, $T$, and follows a time-independent distribution, $p(\tilde{\beta})$. The superstatistical framework has successfully been applied on a widespread of problems like: interactions between hadrons from cosmic rays [4], fluid turbulence [3, 5, 6], granular material [7], electronics [8], economics [9-12], among many others [13]. Furthermore, it has been regarded as a possible foundation for non-extensive statistical mechanics [3] based on Tsallis entropy [14] as we show later on. In this manuscript we introduce a different analysis of differential stochastic dynamics in which the ex-

*Present address: Unilever $R \&$ D, Port Sunlight, Quarry Road East, Bebington, Wirral CH63 3JW, United Kingdom; Electronic address: sdqueiro@ cbpf.br, sdqueiro@googlemail.com ponent of a Feller process is assumed as the superstatistical parameter. The main advantage of this proposal is that it permits the evolution of the functional form of the second-order Kramers-Moyal moment in opposition to previous presentations.

\section{SUPERSTATISTICS}

Consider an inhomogeneous system composed by a large set of cells that have different values of some parameter $\tilde{\beta}$ as we have referred to here above. Within each cell, local equilibrium is reached very promptly. The parameter $\tilde{\beta}$ is taken as constant throughout a period of time $T$ after which it changes into a new value. This update occurs always in accordance with a distribution $p(\tilde{\beta})$ for the parameter. Taking into consideration that each cell is in local equilibrium, thus presenting a Boltzmann factor, $e^{-\beta E},{ }^{1}$ the long-term stationary distribution ${ }^{2}$ of the non-equilibrium system is obtained from a weighted average of local Boltzmann factors, with $\beta \equiv \beta(\tilde{\beta})$,

$$
P(E)=\int p(\tilde{\beta}) \rho(E) \frac{e^{-\beta E}}{Z(\beta)} d \tilde{\beta},
$$

where $\rho(E)$ represents the density of states, and $Z(\beta)$ the normalisation constant. Going back to the example of a Brownian particle moving across a medium treated in Ref. [3], we get that its velocity, $\vec{v}$, is obtained from the local Langevin equation,

$$
d \vec{v}=-\gamma \vec{v} d t+\sigma d \vec{W}_{t}
$$

Seeing that the medium is inhomogeneous, either $\gamma$ [15] or $\sigma$ vary from cell to cell on a large time scale $T^{3}$. Therefore, local Boltzmann factor has,

$$
\beta=\frac{2 \gamma}{m \sigma^{2}}
$$

\footnotetext{
${ }^{1} E$ is the effective energy in each cell.

2 The observation time $t \gg T$.

${ }^{3}$ If $\gamma$ is the random parameter then, $\tilde{\beta}=\gamma$, else if $\sigma$ is the random parameter then, $\tilde{\beta}=\sigma$.
} 
which is random. From Eq. (3), the parameter $\beta$ can also fluctuate for the case of a particle with varying mass [16].

Within time scale $T$, and according to Eq. (2), the local stationary distribution of velocities is a Gaussian conditioned to value $\beta$,

$$
p^{\prime}(\vec{v} \mid \beta)=\left(\frac{\beta}{2 \pi}\right)^{d / 2} \exp \left[-\frac{1}{2} \beta m \vec{v}^{2}\right] .
$$

If the system is able to reach some local equilibrium before an update of $\tilde{\beta}$ takes place, i.e., $T \gg \gamma^{-1}=\tau$, then, we can determine the marginal velocities probability distribution of the long-term behaviour of the Brownian particle,

$$
P(\vec{v})=\int_{0}^{\infty} p(\beta) p^{\prime}(\vec{v} \mid \beta) d \beta
$$

Hence, it is straightforward to verify that the form of $P(\vec{v})$ depends explicitly on the functional form of $p(\beta)$. Specifically, it was verified in Ref. [3] that, when $p(\beta)$ is the $\chi^{2}$-distribution with $n$ degrees of freedom, Eq. (5) yields,

$$
P(\vec{v})=\frac{1}{Z}\left[1+(1-q) \beta_{0} \vec{v}^{2}\right]^{1 /(1-q)},
$$

where $q=1+\frac{2}{n+d}, Z$ is the normalisation factor, and $\beta_{0}$ the average inverse temperature (see Ref. [1] for details). Such a distribution $P(\vec{v})$ maximises Tsallis entropy [14],

$$
S_{q}=\frac{1-\int[p(x)]^{q} d x}{q-1} \quad(q \in \mathfrak{R})
$$

This fact has turned out superstatistics into the first dynamical scenario for the emergence of non-extensive statistical mechanics [17].

\section{THE MODEL}

Consider the following one-dimensional stochastic differential equation,

$$
d v=-\gamma v d t+\omega\left[v^{2}\right]^{\alpha} d W_{t}, \quad(v \neq 0 \quad \text { if } \quad \alpha<0),
$$

where $W_{t}$ is a regular Wiener stochastic process, i.e., $\left\langle d W_{t}\right\rangle=$ 0 , and $\left\langle d W_{t} d W_{t^{\prime}}\right\rangle=d t \delta\left(t-t^{\prime}\right)^{4}$. Stochastic equation (8) belongs to the Feller class of (multiplicative noise) processes [18] with $\gamma \geq 0, \alpha<\frac{1}{4}$ for a (time-dependent) normalisable probability density function (PDF) $f(v, t)$. The associated Fokker-Plank Equation of Eq. (8) is

$$
\frac{\partial f(v, t)}{\partial t}=\frac{\partial}{\partial v}[\gamma v f(v, t)]+\frac{1}{2} \frac{\partial^{2}}{\partial v^{2}}\left[\omega^{2}\left[v^{2}\right]^{2 \alpha} f(v, t)\right]
$$

\footnotetext{
4 The main advantage of writing $\left[v^{2}\right]^{\alpha}$ instead of $|v|^{\alpha^{\prime}}$ with $\alpha^{\prime}=2 \alpha$ is that of analyticity for all $v$ when $\alpha>0$.
}

whose solution $f(v, t)$ relaxes exponentially with a characteristic time, $\tau$, into the stationary solution,

$$
p(v)=\frac{1}{Z} \exp \left[-\frac{\gamma}{\omega^{2}(1-2 \alpha)} v^{2(1-2 \alpha)}\right]\left(v^{2}\right)^{-2 \alpha},
$$

i.e., a Weibull-like distribution, $\mathcal{W}(v)$. $Z$ is the normalisation constant, $\int p(v) d v$,

$$
Z=\frac{2}{1-4 \alpha}\left[\frac{\gamma}{\omega^{2}(1-2 \alpha)}\right]^{\frac{1-4 \alpha}{4 \alpha-2}} \Gamma\left[2+\frac{1}{4 \alpha-2}\right]
$$

For $\alpha=0$, Eq. (8) becomes the standard Langevin equation, and $p(v)$ the Gaussian distribution,

$$
\mathcal{G}(v)=\frac{1}{Z} \exp \left[-\frac{\gamma}{\omega^{2}} v^{2}\right],
$$

with $Z=\sqrt{\frac{\pi \omega^{2}}{\gamma}}$.

After the transient, $f(v, t) \approx p(v)$. The mean value of $v$, $\bar{v} \equiv \int v p(v) d v$, is equal to zero as well as all odd moments of $p(v)$. Regarding the second-order moment, $\overline{v^{2}} \equiv \int v^{2} p(v) d v$, we have got

$$
\overline{v^{2}}=\left\{\begin{array}{cl}
\frac{\omega^{2}}{2 \gamma} & \text { if } \alpha=0 \\
\frac{4 \alpha-1}{4 \alpha-3}\left[\frac{\omega^{2}(1-2 \alpha)}{\gamma}\right]^{\frac{1}{1-2 \alpha}} \frac{\Gamma\left[\frac{5-8 \alpha}{2-4 \alpha}\right]}{\Gamma\left[2+\frac{1}{4 \alpha-2}\right]} & \text { if } \alpha \neq 0
\end{array} .\right.
$$

If we consider $v$ as the velocity of a particle of unitary mass which does a $1 D$ random walk, using equipartition theorem we are able to determine the inverse temperature, $\beta \equiv$ $(k T)^{-15}$, yielding

$$
\beta=\overline{v^{2}}
$$

Evaluating the kurtosis

$$
\kappa \equiv\left(\overline{v^{2}}\right)^{-2} \int v^{4} p(v) d v
$$

we have obtained

$$
\kappa=\left\{\begin{array}{cl}
3 & \text { if } \alpha=0 \\
\frac{(3-4 \alpha)^{2}}{(4 \alpha-5)(4 \alpha-1)} \frac{\Gamma\left[\frac{7-8 \alpha}{2-4 \alpha}\right] \Gamma\left[2+\frac{1}{4 \alpha-2}\right]}{\left\{\Gamma\left[\frac{5-8 \alpha}{2-4 \alpha}\right]\right\}^{2}} & \text { if } \alpha \neq 0
\end{array} .\right.
$$

As it is visible from Fig. 1, distribution (10) is platykurtic for $\alpha<0$, and leptokurtic for $\alpha>0$.

Moving on, we shall now consider that exponent $\alpha$ instead of constant, varies according to superstatistical requirements. In other words, let us consider an inhomogeneous system which is composed by a large set of cells that have different values of $\alpha$. Within each cell, the value of $\alpha$ is updated at

\footnotetext{
${ }^{5}$ In this expression $T$ is the tempetature.
} 

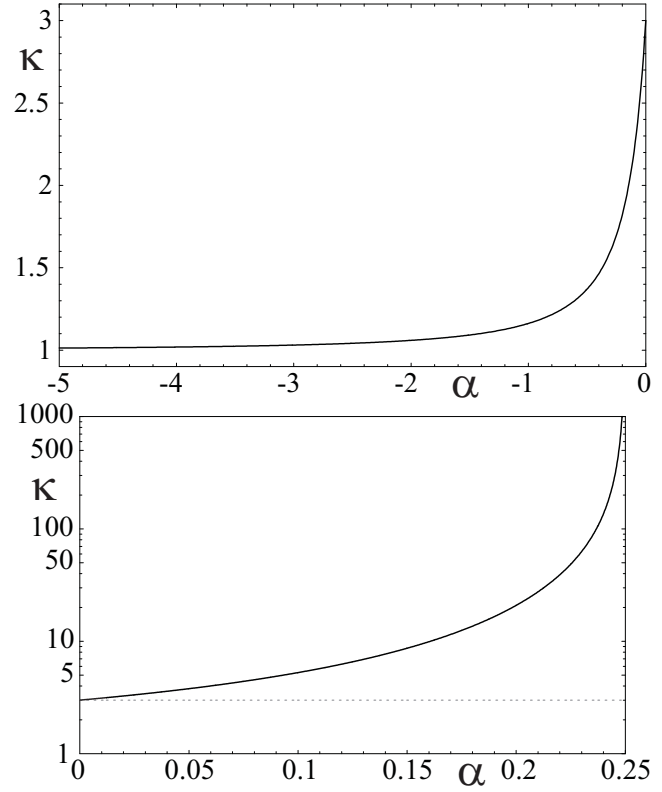

FIG. 1: Kurtosis, $\kappa$, vs. $\alpha$. Upper panel: Non-positive values of $\alpha$ which lead into platykurtic distributions. In the limit $\alpha \rightarrow-\infty$, $\kappa=1$. Lower panel: Non-negative values of $\alpha$ leading into leptokurtic distributions which has $\alpha=\frac{1}{4}$ as upper bound. The dashed line corresponds to the kurtosis of a Gaussian $\kappa=3$.

every elapsed time interval $\ell$, in agreement with a certain PDF $\rho(\alpha)$. The update scale, $\ell$, is much greater than relaxation time scale $\tau$. Noticing that each cell is in local equilibrium, the long-term stationary distribution of the non-equilibrium system is obtained performing the integral,

$$
P(v)=\int \rho(\alpha) p(v) d \alpha .
$$

As possible applications of such a model we name: description of velocities in granular material (particularly see figures of Ref. [19]) and unconventional turbulent fluids, or even the dynamics of financial observables. Explicitly, systems which undergo through different phases during their time evolution or situations where different stages are measured when observations are made at the same point of space. In a thermodynamic context, the fluctuations in $\alpha$ correspond to fluctuations in temperature, but obtained through a completely different way from the proposal presented in Ref. [3]. Specifically, fluctuations in $\alpha$ induce a modification of the functional form of the $2^{\text {nd }}$ order Kramers-Moyal coefficient, while in Ref. [3] its functional form is always preserved.

\section{A. Some examples}

\section{Dichotomous case}

This case represents the simplest form to introduce fluctuations in $\alpha$, and for which a full analytical treatment is possible.
Its probability density function is simply,

$$
\rho(\alpha)=\frac{1}{2} \delta\left(\alpha-\alpha_{0}\right)+\frac{1}{2} \delta\left(\alpha-\alpha_{1}\right), \quad\left(\alpha_{0} \neq \alpha_{1}\right) .
$$

Amongst all endless possibilities for $\alpha_{0}$ and $\alpha_{1}$, let us firstly consider cases for which one of the exponents is equal to zero. The long-term distribution is thus given by

$$
P(v)=\frac{1}{2} \mathcal{G}(x)+\frac{1}{2} \mathcal{W}_{\alpha}(x) .
$$

For small values of $|v|$, and $\alpha<0, P(v)$ approaches the limit $\sqrt{\frac{\gamma}{4 \pi \omega^{2}}}$ as $\left[v^{2}\right]^{-2 \alpha}$.

In Fig. 2 we exhibit the resulting probability density function, $P(v)$, for $\alpha_{0}=0$, and $\alpha_{1}=-1, \alpha_{1}=-\frac{1}{2}$, and $\alpha=\frac{1}{5}$. The value $\overline{v_{e f}^{2}}$,

$$
\overline{v_{e f}^{2}} \equiv \int v^{2} P(v) d v
$$

is presented in Fig. 3 for several values of $\alpha_{1}$.
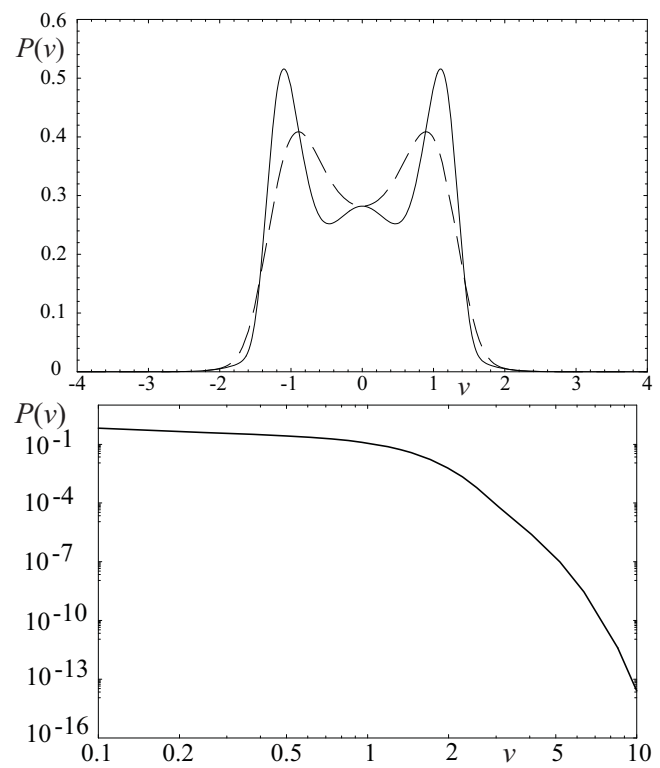

FIG. 2: Probability density function $P(v) v s . \quad v$ for the case of superstatistical processes with a dichotomous distribution (18) where $\gamma=\omega=1$. Upper panel: In both cases $\alpha_{0}=0$, and $\alpha_{1}=-1$ for the full line, and $\alpha_{1}=-\frac{1}{2}$ for the dashed line. Lower panel: $P(v)$ vs. $v$ for a dichotomous case with $\alpha_{0}=0$ and $\alpha=\frac{1}{5}(\gamma=\omega=1)$ in a log$\log$ scale. For this case, the variance $\overline{v_{e f}^{2}}=0.2860 \ldots$ and kurtosis $\kappa=11.953 \ldots$

\section{Uniform distribution case}

If, for the previous example the analytical form of $P(v)$ is easily obtained, that does not happen in situations in which $\alpha$ 

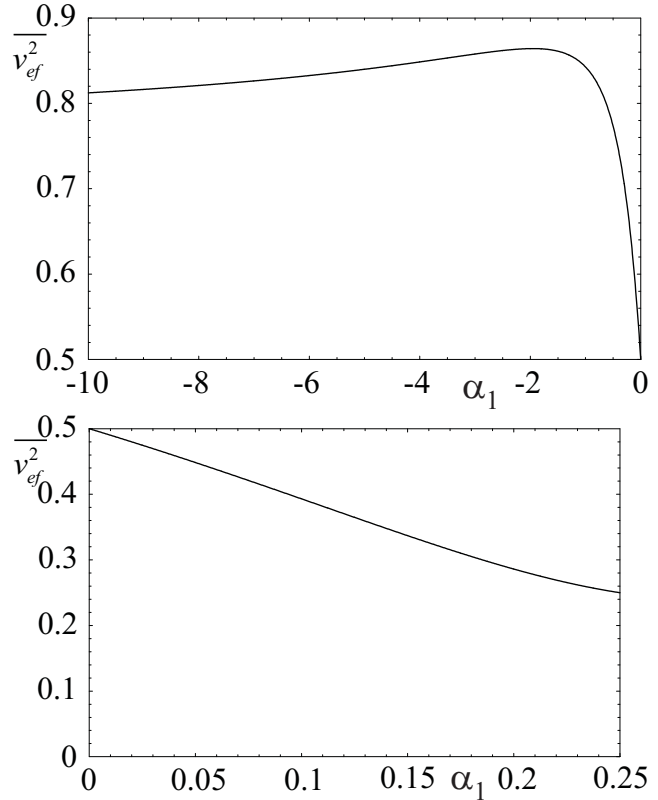

FIG. 3: Standard deviation, $\overline{v_{e f}^{2}}$, vs. dynamical exponent $\alpha_{1}$ for superstatistical process with a dichotomous PDF (18) where $\alpha_{0}=0$, and $\gamma=\omega=1$. For $\alpha_{1}=0, \overline{v_{e f}^{2}}=\frac{1}{2}$, and for $\alpha_{1}=-\infty$ the value of $\overline{v_{e f}^{2}}$ tends to $\frac{3}{4}$ (upper panel). For positive values of $\alpha_{1}, \overline{v_{e f}^{2}}$ is a monotonically decreasing function of $\alpha_{1}$ with $\overline{v_{e f}^{2}}=\frac{1}{4}$ for $\alpha_{1}=\frac{1}{4}$.

evolves according to a uniform distribution between $\alpha_{0}$ e $\alpha_{1}$,

$$
\rho(\alpha)=\left\{\begin{array}{ccc}
\frac{1}{\alpha_{0}-\alpha_{1}} & \text { if } \quad \alpha_{1} \leq \alpha \leq \alpha_{0} \\
0 & \text { otherwise }
\end{array} .\right.
$$

However, it is possible to evaluate numerically the form of the long-term distribution as we present for two particular cases in Fig. 4. Computing the kurtosis, $\kappa$, for both cases we have verified that the two examples are platykurtic.

Another possibility is to consider just non-negative values for $\alpha$. This situation is more likely to be experimentally verified, since kurtosis excess is quite ubiquitous. In this case, and since we are considering leptokurtic distributions, $P(v)$ is also leptokurtic. An illustration of this sort of example is presented in Fig. 5 where $\alpha$ uniformly varies between 0 and $\frac{1}{5}$.

Moreover, extending our range of values for exponent $\alpha$, we can consider positive and negative values as we present in Fig. 6. For this last case, in the long-term, the system endures both platykurtic and leptokurtic regimes.

\section{The $\chi^{2}$-distribution}

Another distribution that appears in various phenomena is the $\chi^{2}$-distribution,

$$
\rho(\alpha)=\frac{v^{v}}{\bar{\alpha} \Gamma[v]}\left(\frac{|\alpha|}{\bar{\alpha}}\right)^{v-1} \exp \left[-\frac{v}{\bar{\alpha}}|\alpha|\right] .
$$

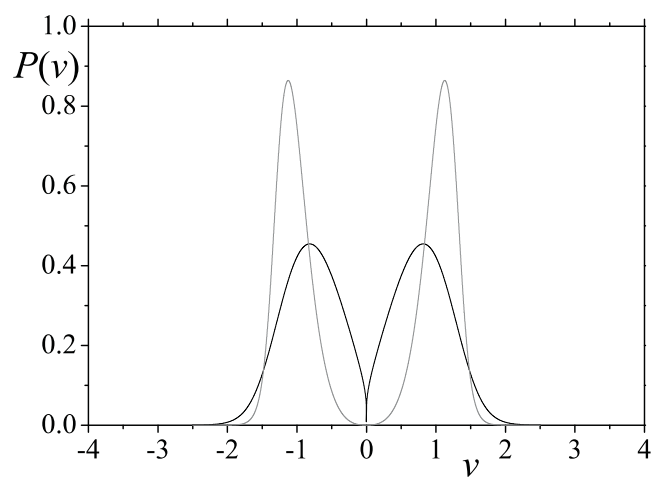

FIG. 4: Numerically obtained probability density function $P(v) v s$. $v$ for the case of superstatistical processes with uniform distribution, Eq. (21) where $\gamma=\omega=1$. The black line corresponds to $\alpha_{0}=0$, and $\alpha_{1}=-\frac{1}{2}$, with $\overline{v_{e f}^{2}}=0.833 \ldots$, and kurtosis, $\kappa=1.755 \ldots$ The grey line corresponds to a uniform distribution with $\alpha_{0}=-\frac{1}{2} \alpha_{1}=-\frac{3}{2}$ yielding $\overline{v_{e f}^{2}}=1.169 \ldots$, and kurtosis, $\kappa=1.179 \ldots$.

For this case an analytical form is not, in principle, possible to obtain. Nonetheless, we obtain the numerical solution for $P(v)$ as we show in Fig. 7 .

\section{B. A generalised Weibull distribution}

In this subsection we treat, the standard case where in Eq. (8) $\omega$ evolves on a superstatistical fashion rather than $\alpha$. For this particular case, when $\Omega \equiv \omega^{-2}$ follows a $\chi^{2}$-distribution,

$$
\rho(\Omega)=\frac{1}{\Omega_{0} \Gamma\left[\frac{v}{2}\right]}\left(\frac{v}{2 \Omega_{0}}\right)^{\frac{v}{2}} \Omega^{2+\frac{v}{2}} \exp \left[-\frac{v}{2} \frac{\Omega}{\Omega_{0}}\right],
$$

the long-term stationary distribution, $P(v)$, that corresponds to a weighted average of $p(v)$ over all possible values of $\Omega$, yields

$$
{ }_{q} \mathcal{W}(v)=\frac{1}{Z^{\prime}} \exp _{q}\left[-\frac{\left(v^{2}\right)^{a}}{\tilde{v}}\right]\left(v^{2}\right)^{b}
$$

where,

$$
q=\frac{4+v-6 \alpha-2 v \alpha}{2+v-2 \alpha-2 v \alpha}, \quad \tilde{v}=\frac{v(1-2 \alpha)^{2} \Omega_{0}}{(2+v-2 \alpha(1+v)) \gamma}
$$

and

$$
a=1-2 \alpha, \quad b=-2 \alpha .
$$

Owing to its comparability to the Weibull distribution inside $S_{q}$ framework, and following Ref. [21] we shall call PDF (24) $q$-Weibull distribution. The $q$-Weibull distribution has been used to numerically adjust a variety of PDFs, but, to the best of my knowledge, no dynamical basis has been presented so far. Distribution (24) belongs to the Burr class of probability density functions [22]. For small values of $|v|,{ }_{q} \mathcal{W}(v)$ in 

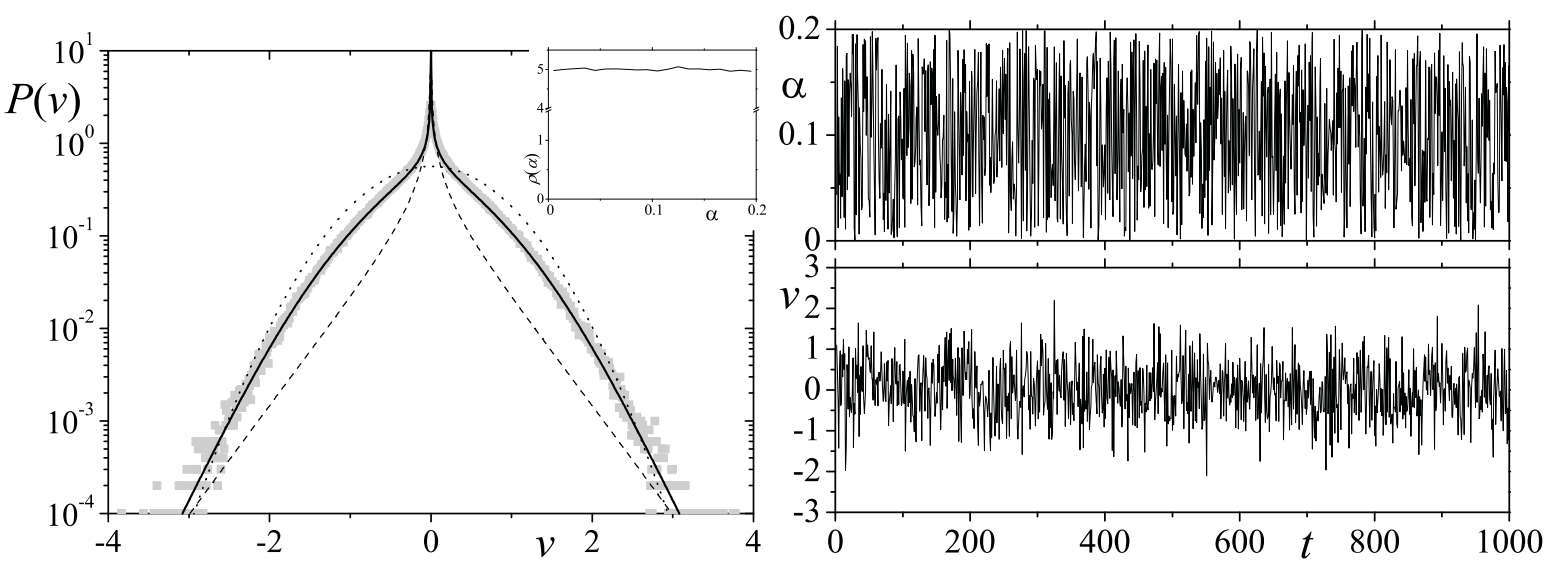

FIG. 5: Left panel: The full line represents the numerically obtained probability density function $P(v) v s . \quad v$ for the case of superstatistical processes with uniform distribution, Eq. (21), where $\alpha_{0}=0$, and $\alpha_{1}=\frac{1}{5}(\gamma=100, \omega=10)$. The dashed line represents the PDF of the upper bound $\alpha=\frac{1}{5}$, and the dotted line the PDF of the lower bound $\alpha=0$ (a Gaussian). The long-term PDF decays slower than the Gaussian. The grey symbols represent the PDF obtained from the numerical simulation of a superstatistical system with the same parameters $\gamma$ and $\omega$ and PDF $\rho(\alpha)$. In the inset we show $\rho(\alpha)$ of that process. Right panels: Excerpt of a superstatistical time series of $v$ which evolves according to Eq. (8) with $\gamma=100, \omega=10$, and $\alpha$ associated with a uniform distribution between $\alpha=0$ and $\alpha=\frac{1}{5}$. The time scale of updating $\alpha$ is 1 time unit which is rather larger than $10^{-2}$ that is the time scale of relaxation towards stationarity. For this case $\alpha_{0}=0$, and $\alpha_{1}=\frac{1}{5}$, with $\overline{v_{e f}^{2}}=0.285 \ldots$, and kurtosis, $\kappa=5.272 \ldots$. A fair similar distribution, namely Fig. 6(b), has been obtained in Ref. [20] for the velocities PDF of a long-range Hamiltonian system at a quasi-stationary state.

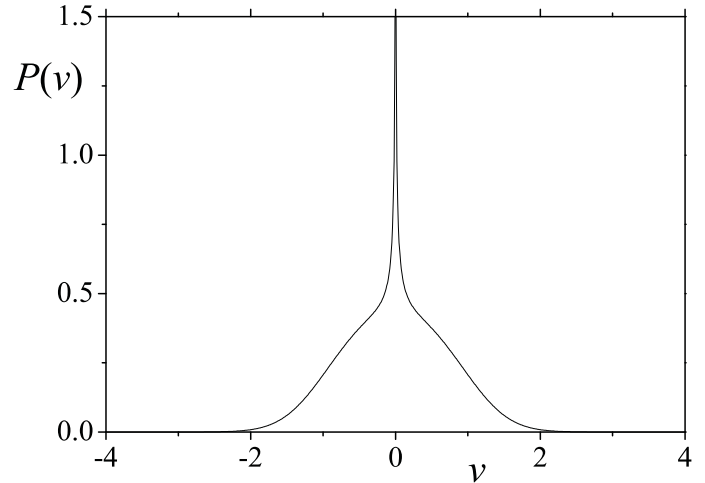

FIG. 6: Numerically obtained probability density function $P(v) v s$. $v$ for the case of superstatistical process with uniform distribution, Eq. (21), where $\alpha_{0}=-\frac{1}{5}$, and $\alpha_{1}=\frac{1}{5}(\gamma=\omega=1)$. In this case $\overline{v_{e f}^{2}}=0.476 \ldots$, and kurtosis, $\kappa=3.129 \ldots$ Interestingly, this example suggests the existence of, at least, one distribution $\rho(\alpha)$ with $\alpha_{0} \neq \alpha_{1} \neq 0$ for which $P(v)$ is mesokurtic.

Eq. (24) goes to zero as a power law with exponent $b$, and for large $|v|$, the same distribution also vanishes as a power law but with exponent $a /(1-q)+b$. For this case, it is possible to evaluate even moments of order $m$ when the following conditions are verified,

$$
a>0, \quad b>-\frac{m+1}{2}, \quad \frac{a}{q-1}-b>\frac{m+1}{2} .
$$

When $b=0$, i.e., $\alpha=0, P(v)$ turns into a $q$-Gaussian distribution recovering the scenario of Ref. [3]. In Fig. 8 we depict

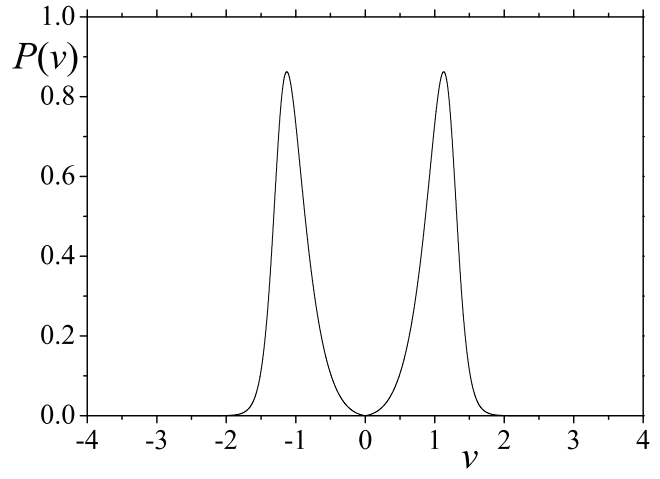

FIG. 7: Numerically obtained probability density function $P(v) v s$. $v$ for the case of superstatistical processes with a $\chi^{2}$-distribution, Eq. (22) where $v=5$, and $\bar{\alpha}=\gamma=\omega=1$. For this example we have $\overline{v_{e f}^{2}}=1.150 \ldots$, and kurtosis, $\kappa=1.999$.

examples of $q \mathcal{W}(v)$ for some values of $a, b$, and $q$.

\section{REMARKS AND PERSPECTIVES}

In this manuscript we have discussed a superstatistical approach of a stochastic process which belongs to the Feller class, and whose (local) stationary probability density function is reminiscent of a Weibull distribution. Such proposal, which might be seen has a dynamical analogue for Ref. [23], has been driven on two ways. The first one in which the power of multiplicative noise term is time-dependent, and another 

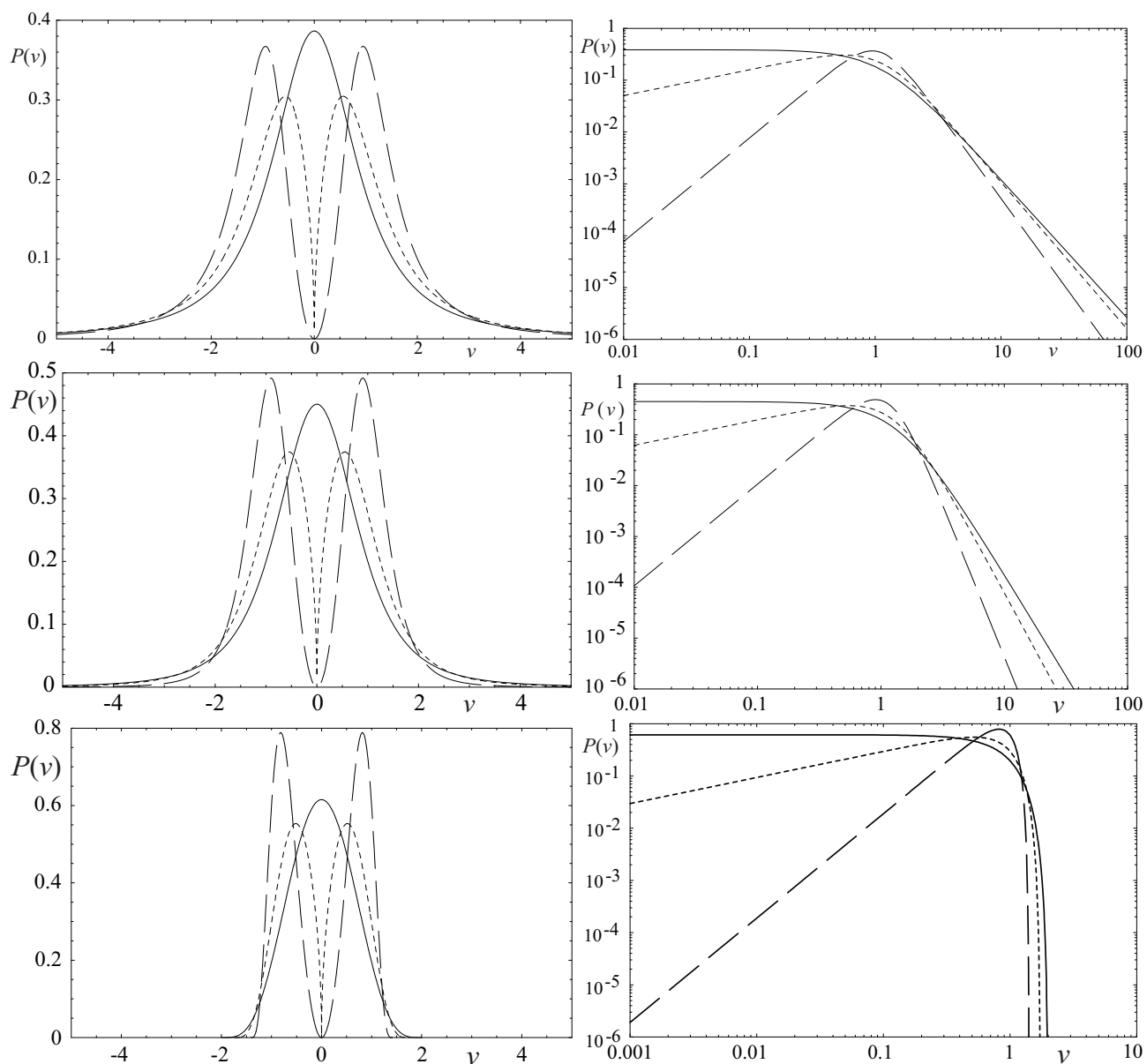

FIG. 8: Representation of ${ }_{q} \mathcal{W}(v) v s$. $v$ for several values of parameters $a, b, q$, and $\tilde{v}=1$. Left panels contain linear-linear representations, whereas right panels exhibit the same ${ }_{q} \mathcal{W}(v)$ but in a log-log scale. For long dashed lines $(a=2, b=1)$; short dashed lines $\left(a=\frac{5}{4}, b=\frac{1}{4}\right)$; and for the full lines $(a=1, b=0)$ which correspond to a $q$-Gaussian PDF. In upper panels we have $q=\frac{7}{4}$. Because of conditions (27), the $(a=2, b=1)$ case is the only one to have a finite $\overline{v_{e f}^{2}}$. Explicitly, $\overline{v_{e f}^{2}}=6.424 \ldots$ Midst panels: $q=\frac{3}{2}$. Both of the three cases have a finite $\overline{v_{e f}^{2}}$. Specifically, $\overline{v_{e f}^{2}}=\sqrt{2}$ for $(a=2, b=1), \overline{v_{e f}^{2}}=2$ for $(a=1, b=0)$, and $\overline{v_{e f}^{2}}=2^{4 / 5}$ for $\left(a=\frac{5}{4}, b=\frac{1}{4}\right)$. Curiously, case $(a=2, b=1)$ has $\kappa=3$ just like a Gaussian distribution, i.e., a mesokurtic distribution. Lower panels: $q=\frac{3}{4}$. In this case, distributions present a compact support, $\left[-v_{c}, v_{c}\right]$, by reason of Tsallis cut-off, where $v_{c}=\left(\frac{1}{1-q}\right)^{\frac{1}{2 a}}$.

one that is equivalent to the evolution of the noise width in the stochastic term of the corresponding differential equation. The main advantage of the former is the possibility of mimicking systems which hold a rather complex dynamics that goes through a (random) sequence of dynamical regimes. We have determined either analytically or numerically the longterm probability density function which can assume all types of kurtosis. Concerning the latter superstatistical approach, we have verified that it allows the emergence of a generalisation of the Weibull distribution within the framework of Tsallis non-additive entropy. This distribution has proved to be valid for numerical adjustments in a large variety of systems. For the $q$-Weibull, we have also been able to obtain platykurtic, mesokurtic, and leptokurtic distributions. In respect of is actual implementation to the analysis of experimental data we must refer that it requires the development of techniques to capture both of $\rho(\alpha)$ and $T$ as it was introduced for cases with fluctuations in $\omega$ [5][24]. The solution for this non-trivial challenge either on an experimental or theoretical level is certainly welcomed.

Last of all, the extension of the idea of a stochastic dynamics with superstatistical exponents that lead to a consistent emergence of leptokurtic long-term probability density functions by means of considering, e.g., a non-zero mean or the combination of independent multiplicative and additive noises will be the focus of further research. 


\section{Acknowledgments}

I am deeply grateful to Professor Constantino Tsallis who has always stimulated my scientific curiosity with his observations, comments, and endless incentive. I thank D. O. SoaresPinto for calling my attention to the work of Ref. [19], and to E.M.F Curado and F.D. Nobre for their ever useful comments. This work benefited from financial support from Fundação para a Ciência e Tecnologia (Portuguese Agency), and also infrastructural support from PRONEX (Brazilian agency). The present piece of work pays homage to Brasil and its flamboy- ant people where and with whom I have had the best times of my life.

Note after acceptance - During a talk given in November 2007 at Queen Mary University of London based on this work I took knowledge by Prof. C. Beck of an article on turbulence, namely, Ref. [25], in which are presented probability density functions with an aspect very similar to some of the distributions presented herein. This underlines the surmised application of the our theoretical model to turbulence models.
[1] C. Beck and E. G. D. Cohen, Physica A 322, 267 (2003).

[2] S. Abe, C. Beck, and E.G.D. Cohen, Phys. Rev. E 76, 031102 (2007).

[3] C. Beck, Phys. Rev. Lett. 87, 180601 (2001).

[4] G. Wilk, Z. Włodarczyk, Phys. Rev. Lett. 84, 2770 (2000).

[5] C. Beck, E. G. D Cohen, and H.L. Swinney, Phys. Rev. E 72, 056133 (2005).

[6] S. Rizzo and A. Rapisarda, Proceedings of the 8th Experimental Chaos Conference, Florence, AIP Conf. Proc. No. 742 (AIP, Melville 200), 176; C. Beck, E. G. D. Cohen, and S. Rizzo, Europhys. News 36, (6) 189 (2005); C. Beck, Phys. Rev. Lett. 98, 064502 (2007)

[7] C. Beck, Physica A 365, 96 (2006).

[8] F. Sattin and L. Salasnich, Phys. Rev. E 65, (R)035106 (2002).

[9] J.P. Bouchaud and M. Potters, Theory of Financial Risks: From Statistical Physics to Risk Management, (Cambridge University Press, Cambridge, 2000); M. Ausloos and K. Ivanova, Phys. Rev. E 68, 046122 (2003).

[10] S. M. Duarte Queirós and C. Tsallis, 2005 Europhys. Lett. 69, 893 (2005); S. M. Duarte Queirós, Physica A 344, 619 (2004); S. M. Duarte Queirós and C. Tsallis, 2005 Eur. Phys. J. B 48, 139 (2005).

[11] S. M. Duarte Queirós, Europhys. Lett. 71, 339 (2005).

[12] J. de Souza, L. G. Moyano, and S.M. Duarte Queirós, Eur. Phys. J. B 50, 165 (2006).

[13] C. Beck, Europhys. Lett. 64, 151 (2003); A. M. Reynolds, Phys.
Rev. Lett. 91, 084503 (2003); N. Mordant, A. M. Crawford, and E. Bodenschatz, Physica D 193, 245 (2004); S. Jung and H. L. Swinney, Phys. Rev. E 72, 026304 (2005); C. Beck, Physica D 193, 195 (2004); K. E. Daniels, C. Beck, and E. Bodenschatz, Physica D 193, 208 (2004); C. Beck, Physica A 331, 173 (2004); S. Abe and S. Thurner, Phys. Rev. E 72, 036102 (2005).

[14] C. Tsallis, J. Stat. Phys. 52, 479 (1988).

[15] J. Łuczka, P. Talkner, and P. Hänggi, Physica A 278, 18 (2000).

[16] M. Ausloos and R. Lambiotte, Phys. Rev. E 73, 011105 (2006).

[17] E. G. D. Cohen, Pramana, J. Phys. 64, 635 (2005).

[18] W. Feller, Ann. Math. 54, 173 (1951).

[19] W. A. M. Morgado and E. R. Mucciolo, Physica A 311, 150 (2002).

[20] A. Pluchino, A. Rapisarda, and C. Tsallis, EPL 80, 26002 (2007).

[21] S. Picoli Jr., R. S. Mendes, and L. C. Malacarne, Physica A 324, 678 (2003).

[22] I. W. Burr, Ann. Math. Stat. 13, 215 (1942); S. Nadarajah and S. Kotz, Physica A 377, 465 (2007).

[23] G. A. Tsekouras and C. Tsallis, Phys. Rev. E 71, 046144 (2005).

[24] S. M. Duarte Queirós, Physica A 385, 191 (2007).

[25] B. Castaing, Y. Gagne, and H. J. Hopfinger, Physica D 46, 150 (1990). 